\title{
Assessment of Knowledge Regarding Hospital Acquired Infections (Nosocomial Infection) Among Health Care Workers in a Tertiary Care Hospital of Wah Cantt
}

\author{
${\text { Atika } \text { Akram }^{1 *} \text {, Sana Majeed }}^{1}$, Habib-Ur-Rehman ${ }^{2}$ \\ ${ }^{1}$ Nursing Lecturer at Institute of Nursing Wah Medical College, North Road, Wah, Rawalpindi, Punjab, Pakistan \\ ${ }^{2}$ Nursing Lecturer at Rawal College of Nursing, Sanam Chowk, Khanna, Khanna, Islamabad, Islamabad Capital Territory, Pakistan
}

DOI: $10.36348 /$ sjnhc.2020.v03i02.007
*Corresponding author: Atika Akram

Objective: To assess the knowledge regarding hospital acquired infection (nosocomial infection) among health care workers of tertiary care hospital of Wah Cantt. Study Design: This was a Descriptive quantitative study design based on self-design questionnaire. Place and Study Duration: It was carried out from March 2018 to August 2018 involving health care workers working in tertiary care hospital of Wah Cantt. Materials and Methods: Data was collected through self-structured questionnaire from the health care workers working in tertiary care hospital of Wah Cantt. The collected data was analyzed through SPSS version 20, for proper analysis the data was presented in the form of percentage and frequencies for qualitative variables, mean and standard deviation was calculated for quantitative variables. Results: The results of the study showed that Health Care Workers had adequate knowledge regarding hospital acquired infection. The existing studies was done to explore the knowledge gap regarding hospital-acquired infection of Health Care Workers and how hospital acquired infection can prevent from one another in the hospital settings. Conclusion: The study gave a basic level of knowledge regarding hospital acquired infection (nosocomial infection) among health care workers. The health care associated infection (HCAIs) or the infections acquired from the hospital can be defined as the Infection, which occurs within the 48 hours of hospital admission or three days of discharge from hospital.

Keywords: Knowledge, Hospital Acquired Infection, Health Care Workers, Nosocomial Infection.

Copyright @ 2020: This is an open-access article distributed under the terms of the Creative Commons Attribution license which permits unrestricted
use, distribution, and reproduction in any medium for non-commercial use (NonCommercial, or CC-BY-NC) provided the original author and source
are credited.

\section{INTRODUCTION}

Nosocomial infections (Hospital Acquired Infections) are all those infections which develops 48 hours after admission to the hospital or within 48 hours after discharge from the hospital that was not present at the time of admission to hospital [1].

Hospital acquired or health care associated infections are previously known as nosocomial infections. Patients come in hospital for treatment and early management but they also gain some other serious infections from health care staffs. These types of infections occur in children, adults and also in old age people. Respiratory tract infections are more common in children and urinary tract infections are in adults. A well organised infection control programme can prevent $25-50 \%$ of HAI as stated by the "Hospital Infection Society of India" [2].
The patients like infants in NICU, who require the most therapeutic interventions to support them leading to frequent invasive procedures and the longest exposure to the hospital environment are most affected by hospital acquired infections. However, infection rates vary from one unit to another, suggesting that there are differences in either how infection rates are determined or the care provided in the various units [3].

A study conducted in Ethiopia among 150 health care workers working in a hospital, systematic random sampling technique was used to identify factors for knowledge and practices of infection prevention. The study results showed that health care workers had good knowledge of infection prevention but they had inadequate practices regarding infection prevention. $84 \%$ participants had good knowledge, factors associated were their educational level, sex, age, working experience and even training of infection control. The results showed that MSc and BSc level nurses had good practices than diploma level nurses. It 
also revealed that work experience had strong impact on practices of nurses regarding infection control. Hospital acquired infection is one of the major global burden on health. Health care workers play major role in prevention of infection from themselves and their patients also [4].

In Qatar Ibrahim was conducted a crosssectional interview-based survey to evaluate knowledge and attitude regarding infection control among medical students. 73 participants were included in the study. $48.4 \%$ had adequate knowledge about standard isolation precautions, $85 \%$ participants had knowledge regarding hand hygiene practice. The study results showed that minimal hand hygiene training was given to the medical students and only $35 \%$ senior medical doctors follow the standard precautions [5].

Another study conducted in Nigeria regarding infection control knowledge and practices among HCWs, 200 (doctors and nurses) participants were included in the study. $87 \%$ respondents identified that hand hygiene was the most effective barrier to control hospital acquired infections. Nurses had better knowledge regarding infection control than doctors. Few participants had knowledge about the risks of infection. $56 \%$ doctors and $76 \%$ nurses practiced hand hygiene. The study results showed that there was gap between knowledge and practices regarding infection control. They had good knowledge but their practices were not good regarding standard isolation precautions and hand hygiene [6].

Another study conducted in India about knowledge, attitude and practices regarding nosocomial infection, 150 health care workers were included in the study. The study showed that many nosocomial infections were transferred via direct contact because health care workers didn't wash their hands before and after any procedure. Many barriers were observed such as limited use of hand disinfection and glove use. There were multiple reasons for compliance of hand washing among different health care workers such as dryness of skin, shortage of staff and resources, shortage of time and work burden [7].

Health care infections were the leading cause of hospital acquired infections in Pakistan and in developing countries. A study conducted in Pakistan about knowledge, attitude and practices regarding infection control among HCWs. 198 participants were included in the study. The study results showed that $86 \%$ doctors had good knowledge, nurses had $76 \%$ knowledge and paramedical staffs had $73 \%$ knowledge regarding infection control. It also showed that doctors had better attitude towards infection prevention than other health care workers. Continue education program was needed to prevent hospital acquired infections and it was necessary for practicing standard precautions [8].

\section{MATERIALS AND METHODS}

This was a descriptive quantitative study design based on self-design questionnaire carried out from March 2018 to August 2018 involving health care workers working in tertiary care hospital of Wah Cantt. Non-probability / non-random (convenient sampling) was carried out among doctors, nurses and technicians who were in direct contact with the patient. Sweepers, ayas, transporters who were not in direct contact with the patient, were not included in the study. Informed consent was signed and purpose of the study was explained to the all participants. All participants become the part of this study on their willingness. Confidentiality was ensured to protect the ethical right of all participants.

\section{RESULTS}

Hospital acquired infections were the most common infection that patients got during their stay at hospital, before and after treatment or any procedure they acquired infections. This was a serious problem in health care settings that HAIs were the most common and dangerous causes of illness and death. Health care workers were the main source who spread infections rapidly from one patient to another.

It was difficult to mention all departments separately, so we divided all departments in two categories,
A. Critical area
B. Non-critical area

\begin{tabular}{|c|c|c|}
\hline Departments & Frequency & Percent \\
\hline Critical area & 29 & 58.0 \\
\hline Non-Critical area & 21 & 42.0 \\
\hline Total & 50 & 100.0 \\
\hline
\end{tabular}

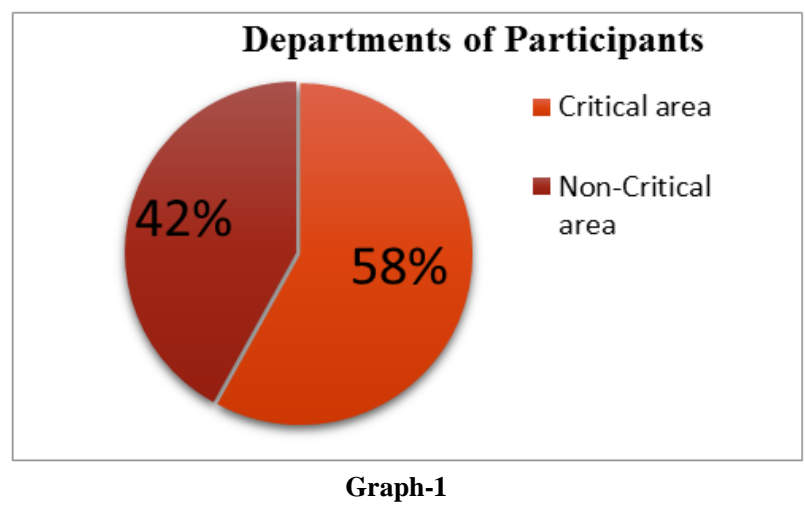

$58 \%$ HCW's participated from Critical area while $42 \%$ participated from Non-Critical area. 


\section{PARTICIPANTS}

Table-2

\begin{tabular}{|l|l|l|}
\hline Participants & Frequency & Percent \\
\hline Doctors & 11 & 22.0 \\
\hline Nurses & 29 & 58.0 \\
\hline Nursing Assistant & 2 & 4.0 \\
\hline Radiologist & 1 & 2.0 \\
\hline Pharmacist & 5 & 10.0 \\
\hline Others & 2 & 4.0 \\
\hline Total & 50 & 100.0 \\
\hline
\end{tabular}

\section{Participants}

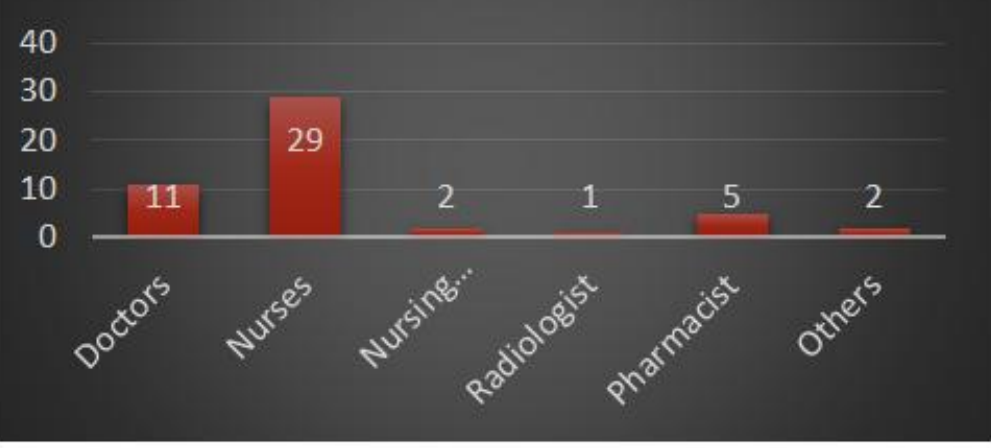

Graph-2

In our study we selected these professionals who were directly concerned with patients i.e. Doctors, Nurses, Nursing-Assistant, Radiologists, Pharmacists and others (OT technicians and ICU/ER technicians).
After simple random sampling, 29 Nurses, 11 Doctors, 5 Pharmacists and 5 were other respondents were included in the study.

We assessed knowledge through knowledge assessment scale

\begin{tabular}{|l|l|}
\hline Knowledge level & Interpretation \\
\hline Good Knowledge & If the respondents give $60-80 \%$ correct answers from the structured questionnaire \\
\hline Adequate Knowledge & If the respondents give 50\% - 60\% correct answers from the structured questionnaire. \\
\hline Poor Knowledge & If the respondents give $<50 \%$ correct answers from the structured questionnaire. \\
\hline
\end{tabular}

Table-3: Knowledge level of Participants Regarding HAI

\begin{tabular}{|l|l|l|}
\hline $\begin{array}{l}\text { Knowledge level of } \\
\text { Participants }\end{array}$ & Frequency & Percent \\
\hline Poor & 4 & 8.0 \\
\hline Adequate & 40 & 80.0 \\
\hline Good & 6 & 12.0 \\
\hline Total & 50 & 100.0 \\
\hline
\end{tabular}

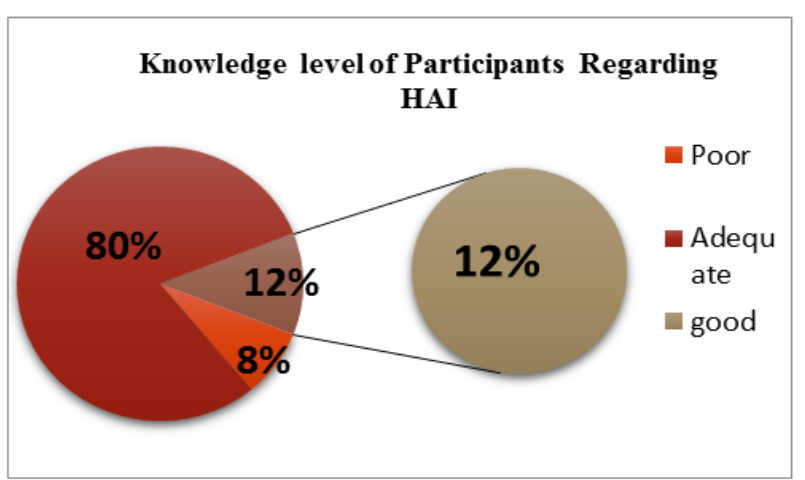

Graph-3: Knowledge level of Participants Regarding HAI 
According to Table-3 majority of HCWs had adequate level knowledge that was $80 \%$. Poor knowledge level of HCWs was $08 \%$, and good level containing HCWs was only $12 \%$. This percentage was represented graphically in Graph-3. They had adequate knowledge about nosocomial infections. Co-worker had very little knowledge about nosocomial infections.

Table-4: Source of Information Regarding HAI

\begin{tabular}{|l|l|l|}
\hline Source of Information & Frequency & Percent \\
\hline Scientific journals & 6 & 12.0 \\
\hline Mass-media & 11 & 22.0 \\
\hline Educational Courses & 31 & 62.0 \\
\hline Physicians & 1 & 2.0 \\
\hline Others & 1 & 2.0 \\
\hline Total & 50 & 100.0 \\
\hline
\end{tabular}

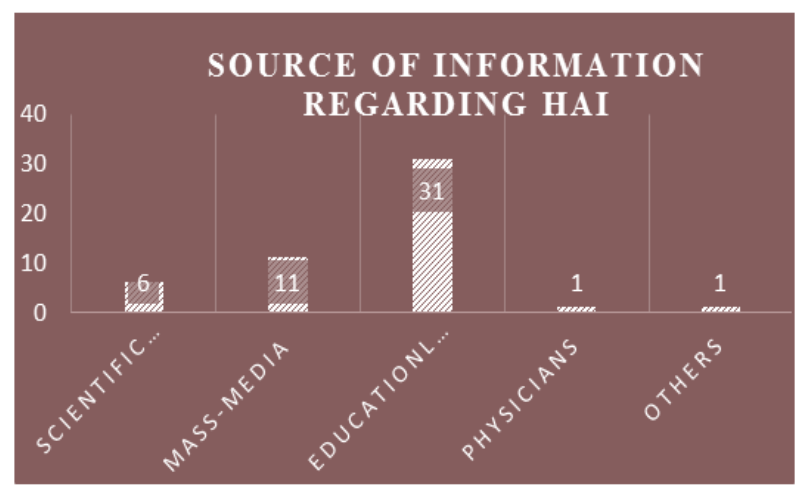

Graph-4: Source of Information Regarding HAI

The study results showed that the major source of information about HAI was educational courses and some respondents also got information about nosocomial infections through other sources as media and others.

\section{DISCUSSION}

However, the importance of gaining support from all levels, (e.g. front staff, unit leadership and Management) just for no resistance to the study implementation, an unfortunate obstacle that became difficult to overcome. This study was conducted to know the knowledge level of Health care workers about Hospital Acquired Infections. In this study all the HCWs (i.e. Doctors, Nurses, Pharmacists, Technicians, etc.) were participated interestedly and gave their response actively. This study was already conducted nationally and internationally to know the knowledge level of HCWs about HIA. Nelia Bruce (University of Massachusetts, Amherst in May 2013) conducted a similar study.

In this study, the goal was to assist the organization to understand why staff members were selected to follow or not to follow the guidelines relating to HAI prevention. Although it has been traditionally suggested that knowledge is often the primary motivator in the process of change, the results of this improvement project highlighted that knowledge and belief related to HAIs is not the deficit as almost all participants expressed that their patients were susceptible to HAI acquisition and they could impact patient outcomes.

A study conducted in Ethiopia among 150 health care workers working in a hospital, 84\% participants had good knowledge, factors associated were their educational level, sex, age, working experience and even training of infection control. It revealed that work experience had strong impact on practices of nurses regarding infection control. Hospital acquired infection is one of the major global burden on health.

In our study only $12 \%$ participants had good knowledge and $80 \%$ majority of the respondents had adequate level knowledge. The study results revealed that improvement in the knowledge, attitude and practices towards nosocomial infection need to be done. For this purpose, continuing education programs, seminars, symposiums or workshops should be arranged on regular basis. There should be proper infection control team.

In Qatar Ibrahim was conducted a crosssectional interview-based survey to evaluate knowledge and attitude regarding infection control among medical students. $48.4 \%$ had adequate knowledge about standard isolation precautions, $85 \%$ participants had knowledge regarding hand hygiene practice. The study results showed that minimal hand hygiene training was given to the medical students and only $35 \%$ senior medical doctors follow the standard precautions [5].

The outcome of this study may be beneficial for health care workers to know the importance of knowledge regarding hospital acquired infection for the prevention of Hospital Acquired Infection by using standard percussion. Knowledge regarding hospital acquired infection can improve the quality outcome of treatment of disease resulting in can improve patient health status. This knowledge will be helpful to improve the work quality of health care workers. This will prevent the patient from hospital acquired infection.

\section{CONCLUSION}

Hospital acquired or health care associated infections are previously known as nosocomial infections. These infections which develop in 48 hours after admission to the hospital or within 48 hours after discharge from the hospital. Health care workers are the main source of HAIs. There are many reasons such as increasing inpatient activity of illness, inadequate nurse patient staffing ratios, unavailability of system resources and other demands have challenged heath care providers. Preventions of nosocomial infection is the responsibility of all individuals and health care providers. For the promotion of good health care 
appropriate isolation, sterilization, other practices and adequate staff training should be necessary. Hospital must provide sufficient resources to support this programme.

\section{RECOMMENDATIONS}

Proper Infection Control team should be arranged to look the Health Care Workers for hand washing as often as possible, use of alcoholic swab, hand scrub, cleaning stethoscope daily basis with alcohol swab, wearing gloves, intravenous catheterization through disinfection of skin before insertion and other clinical practices. The knowledge about HAI improves with regular classes, seminars and different practical activities. Through all these preventing measures, we can reduce the risk of Hospital Acquired infection. Additional concern should be given to critical areas. Further studies are essential in the evaluation of the situation of infection control indifferent areas in hospitals. More intensive and regular training programs to all health care workers must be included in the plans of quality control in the hospital. Regular inspection and follow-up from the ministry of health guarantees good infection control practices.

\section{REFERENCES}

1. Shaikh, N., Morone, N. E., Bost, J. E., \& Farrell, M. H. (2008). Prevalence of urinary tract infection in childhood: a meta-analysis. The Pediatric infectious disease journal, 27(4), 302-308.

2. Revelas, A. (2012). Healthcare-associated infections: A public health problem. Nigerian medical journal: journal of the Nigeria Medical Association, 53(2), 59.
3. Kouzegaran, S., \& Mohammadzadeh, A. (2013). Assessment of Knowledge of the Medical staff (Residents and Nurses) In Nosocomial infections in NICU in Mashhad. Iranian Journal of Neonatology IJN, 3, 1-1.

4. Desta, M., Ayenew, T., Sitotaw, N., Tegegne, N., Dires, M., \& Getie, M. (2018). Knowledge, practice and associated factors of infection prevention among healthcare workers in Debre Markos referral hospital, Northwest Ethiopia. BMC health services research,18(1), 465.

5. Ibrahim, A. A., \& Elshafie, S. S. (2016). Knowledge, awareness, and attitude regarding infection prevention and control among medical students: a call for educational intervention. Advances in medical education and practice, 7,505 .

6. Iliyasu, G., Dayyab, F. M., Habib, Z. G., Tiamiyu, A. B., Abubakar, S., Mijinyawa, M. S., \& Habib, A. G. (2016). Knowledge and practices of infection control among healthcare workers in a Tertiary Referral Center in North-Western Nigeria. Annals of African medicine, 15(1), 34.

7. Suchitra, J. B., \& Devi, N. L. (2007). Impact of education on knowledge, attitudes and practices among various categories of health care workers on nosocomial infections. Indian journal of medical microbiology, 25(3), 181.

8. Khan, M., \& Ishaq, M. (2018). Knowledge, Attitude and Practices regarding infection control among Healthcare Professional at Saidu Teaching Hospital (STH), Swat, Khyber Pukhtoon Khwa, Pakistan, 2015. International Journal of Infectious Diseases, 73, 263. 